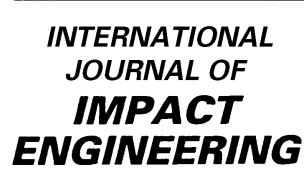

PERGAMON

International Journal of Impact Engineering 24 (2000) 685-701

www.elsevier.com/locate/ijimpeng

\title{
Damage evolution, localization and failure of solids subjected to impact loading
}

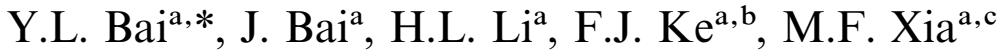 \\ ${ }^{a}$ Laboratory for Non-linear Mechanics of Continuous Media, Institute of Mechanics, Chinese Academy of Sciences, \\ Beijing 100080, People's Republic of China \\ ${ }^{\mathrm{b}}$ Department of Applied Physics, Beijing University of Aeronautics and Astronautics, Beijing 100083, \\ People's Republic of China \\ ${ }^{\mathrm{c}}$ Department of Physics, Peking University, Beijing 100871, People's Republic of China
}

Received 18 April 1999; received in revised form 6 August 1999

\begin{abstract}
In order to reveal the underlying mesoscopic mechanism governing the experimentally observed failure in solids subjected to impact loading, this paper presents a model of statistical microdamage evolution to macroscopic failure, in particular to spallation. Based on statistical microdamage mechanics and experimental measurement of nucleation and growth of microcracks in an Al alloy subjected to plate impact loading, the evolution law of damage and the dynamical function of damage are obtained. Then, a lower bound to damage localization can be derived. It is found that the damage evolution beyond the threshold of damage localization is extremely fast. So, damage localization can serve as a precursor to failure. This is supported by experimental observations. On the other hand, the prediction of failure becomes more accurate, when the dynamic function of damage is fitted with longer experimental observations. We also looked at the failure in creep with the same idea. Still, damage localization is a nice precursor to failure in creep rupture. (C) 2000 Elsevier Science Ltd. All rights reserved.
\end{abstract}

Keywords: Spallation; Damage evolution; Dynamic function of damage; Damage localization; Failure

\section{Introduction}

When a large crack in a solid is predominate, fracture mechanics (linear elastic or elastic plastic) are very successful to characterise the mechanical behaviour of the solid. However, for most

* Corresponding author.

E-mail address: baiyl@lnm.imech.ac.cn (Y.L. Bai). 


\section{Nomenclature}

$\begin{array}{ll}A & \text { average growth rate of microdamage } \\ c & \text { current size of microdamage } \\ c_{0} & \text { initial size of microdamage } \\ C=\dot{c} & \text { growth rate of microcracks } \\ D & \text { continum damage } \\ f & \text { dynamic function of damage (DFD) } \\ k & \text { module of Weibull distribution } \\ m & \text { index of compound damage in DFD } \\ n & \text { number density of microdamage in phase space } \\ n_{0} & \text { number density of microcracks in phase space }\left(c, c_{0}\right) \\ n_{\mathrm{A}} & \text { annihilation rate density of microdamage } \\ n_{\mathrm{N}} & \text { nucleation rate density of microdamage } \\ n_{\mathrm{S}} & \text { number density of microdamage on sectional surface } \\ N(c) & \text { cumulated number density of microdamage greater than } c \\ p_{\mathrm{i}} & \text { independent variables describing the state of microdamage } \\ P_{i}=\dot{p}_{i} & \text { rates of variables } p_{i} \\ t & \text { time } \\ T & \text { time in Lagrangian coordinate } \\ V & \text { current specific volume } \\ V_{0} & \text { initial specific volume } \\ v & \text { particle velocity } \\ \mathbf{x} & \text { macroscopic spatial coordinates of the element where microdamages locate } \\ \mathrm{Y} & \text { spatial coordinate in Lagrangian coordinate }\end{array}$

\section{Greek letters}

$\beta \quad$ intrinsic Deborah number in DFD

$\mu \quad$ parameter in growth rate of microcracks

$\sigma \quad$ nominal stress

$\tau \quad$ average failure volume of a microdamage

$\dot{\theta} \quad$ dilatation rate

Subscripts

$\mathrm{s} \quad$ parameters on sectional surface

c critical values

\section{Superscripts}

$\begin{array}{ll}* & \text { characteristic parameters } \\ \text {. } & \text { rates of variables }\end{array}$


heterogeneous materials, such as alloys, ceramics, composites, etc., there might be distributed microcracks or microvoids rather than a single macroscopical crack. In particular, it is well known that the distributed microdamages usually dominate dynamic failure of solids under impact loading, like spallation under plate impact. For example, the particulates in alloys can become the sites of microdamages in spallation. Owing to the rate-dependent nature of spallation there have been various efforts to formulate this process, such as the integral criterion [1], continuum measure of spallation [2], microstatistical fracture mechanics [3], etc. All these approaches provide progressively helpful means to reveal the essence of this rate-dependent failure process. In particular, Curran et al. [3] gave a sophisticated description of the microscopic processes and their link to continuum fracture mechanics. Meyers has provided a very comprehensive overview on various aspects of spalling [4]. In this overview, he stressed on two aspects. These are the quantitative/predictive models based on continuum measure of spalling and nucleation-andgrowth of microcracks and the utmost important microstructural effects on spalling. Clearly, these offer us a real challenge.

In recent $10 \mathrm{yr}$, some new and informative studies relevant to spallation are made to have deeper understanding of the process. These are the study of microscopic ductile rupture [5], the ultrasonic imaging of spall damage under repeated plate impact with C-scan [6], the interpretation of integral criterion for spallation in terms of damage measure defined by residual strength [7], crack straining spall model [8] and a model on crack population [9]. All these works intended to link the macroscopic spallation and the microdamage evolution inside the materials. This clearly demonstrates that there is still an acute lack of quantitative/predictive models based on the dynamics of nucleation-and-growth of microcracks and their continuum measure in the study of spallation, as reviewed by Meyers [4].

Then, what is the alternative to fracture mechanics, continuum damage mechanics or other previous approaches to deal with the problem. As mentioned above that the accumulation of microdamages has significant effect on the failure of materials, like spallation. Roughly speaking, microdamages are in the size of particulates or grains, like micrometres, and the total of such microdamages on the surface of metals is in the range of $10^{2}-10^{4} / \mathrm{mm}^{2},[10,11]$. So, the main issues in microdamage evolution are nucleation, growth and coalescence of microdamages $[3,4,10,11]$. This means that a trans-scale (from meso- to macro-scopic) understanding of damage evolution is badly needed.

For this sake, a fundamental equation of microdamage evolution has been established in the light of statistical mesoscopic damage mechanics, [12,13]. This is the evolution equation of microdamage in phase space

$$
\frac{\partial n}{\partial t}+\sum_{i=1}^{I} \frac{\partial\left(n \cdot P_{i}\right)}{\partial p_{i}}=n_{\mathrm{N}}-n_{\mathrm{A}}
$$

where $n$ is the number density of microdamage in phase space and $t$ is time. $p_{i}$ are the independent variables describing the state of microdamages. For example, $p_{i}$ can be the current size of microdamage $c$, the initial size of microdamage $c_{0}$, the macroscopic spatial coordinates $\mathbf{x}$ of the element where the microdamage locates, etc., $P_{i}=\dot{p}_{i}$ are the rates of variables $p_{i} . n_{\mathrm{N}}$ and $n_{\mathrm{A}}$ are nucleation and annihilation rate densities of microdamage, respectively. 
It has also been found that the population of microdamages has a tendency to form macroscopically localized damage, a precursor to failure. This can be linked to a special feature of the dynamic function of damage, which is a macroscopic representation of the nucleation, growth and coalescence of microdamages $[14,15]$.

According to the above-mentioned knowledge, in this paper we intend to reveal the link between mesoscopic mechanisms and macroscopic formulations of spallation in terms of the experimental observations. Then, we shall provide some tool for the prediction of the dynamic failure.

\section{Outline of experimental observations of microcrack evolution}

For experimental observation of microcrack evolution in spallation, we use one stage light gas gun to establish a uniaxial strain state in the specimen. The testing material we used is an aluminium alloy similar to 2219-T6 $\mathrm{Al}$ (with $6 \% \mathrm{Cu}$, etc.), for more details see [16]. A short stress pulse of about $0.1 \mu$ s duration was used to investigate the nucleation [10] and a multi-stress pulse technique to study the successive nucleation, growth and coalescence of microcracks [11]. A case study of microdamage evolution in the Al alloy was carried out with the multi-stress pulse technique under impact velocity of $172 \mathrm{~m} / \mathrm{s}$, corresponding to about $1470 \mathrm{MPa}$ tensile stress, [11]. In all these studies, we combined the transient recording of stresses and the counting of microdamages in specimens after recovery.

In order to investigate the evolution of microcracks, proper counting of microcracks in tested specimens is a key step. After impact, specimens should be carefully recovered by a specially designed catcher in the gun to prevent secondary damage. Then the tested specimens were sectioned and polished carefully. Microscopic observations and counting of microcracks were conducted with an S-570 scanning electron microscope and a Q-520 image analysis system with Polyvar-Met microscope. The details of the counting procedure can be referred to $[10,11]$. Fig. 1 shows a typical microscopic picture of microdamages on the section of an impacted specimen.

The followings are some main points of experimental observations of microcracks in the impacted Al alloy $[10,11]$.

1. The nucleation size distribution of microcracks on the sectional surface of specimen can be fitted to Weibull distribution, similar to the size distribution of second phase particles in the specimen

$$
\left(\frac{c_{0}}{c^{*}}\right)^{k-1} \exp \left[-\left(\frac{c_{0}}{c^{*}}\right)^{k}\right],
$$

where $c_{0}$ is the size of microcracks at nucleation and $c^{*}=4.27 \mu \mathrm{m}$ is a characteristic size of the material, roughly the size of the second-phase particles of the alloy. Fitting parameter $k$ is Weibull module and $k=2.33$.

2. The total of microcracks under constant tensile stress appears to be proportional to loading duration. The rate of nucleation on sectional surface $N_{\mathrm{N}, \mathrm{s}}$ appears to be independent of existing microcracks and its average is about $928 /\left(\mathrm{mm}^{2} \mu \mathrm{s}\right)$ under tensile stress of $1470 \mathrm{MPa}$. 


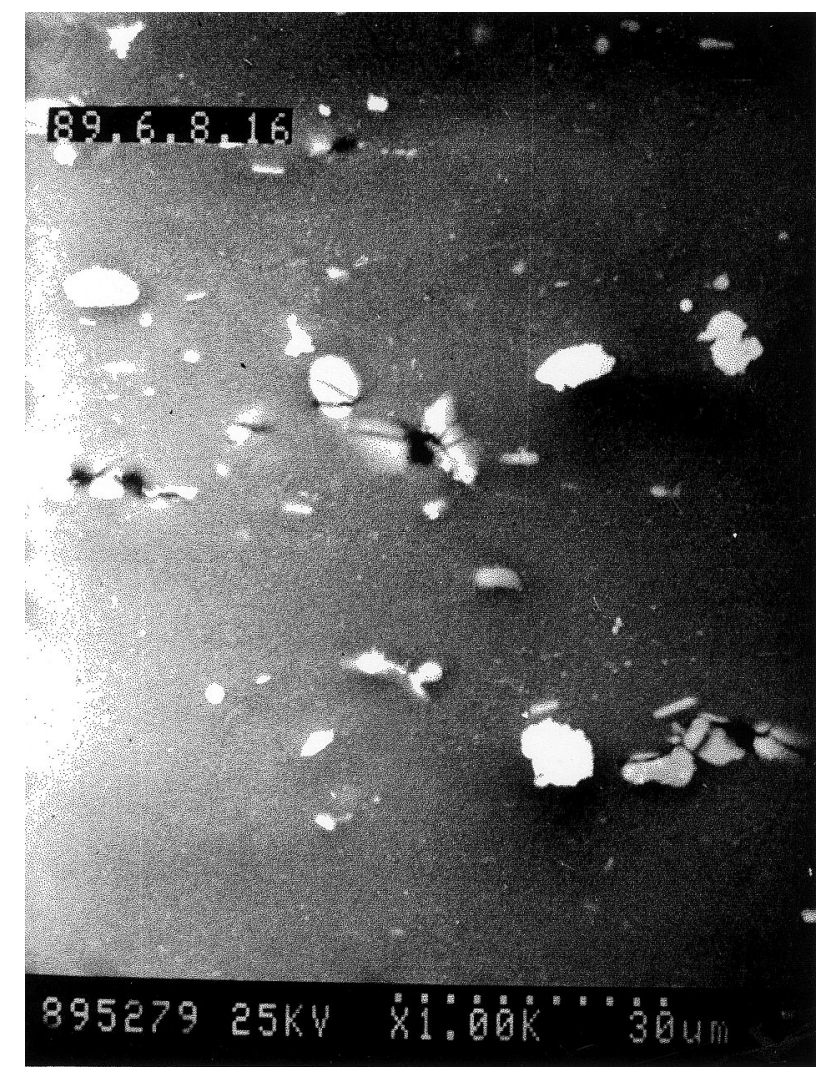

Fig. 1. Microcracks on the section of an impacted aluminium alloy specimen (impact velocity: $593 \mathrm{~m} / \mathrm{s}$, tensile stress: $7460 \mathrm{MPa}$, time duration: $142.2 \mathrm{~ns})$.

3. The nucleation rate of microcracks can be approximately correlated to tensile stress by a linear function, i.e.

$$
\left(\frac{\sigma}{\sigma^{*}}-1\right)
$$

where $\sigma$ is nominal stress and $\sigma^{*}=450 \mathrm{MPa}$ is the threshold of nucleation of microcracks, respectively.

4. Above all, the nucleation rate of microcracks on sectional surface can be expressed by

$$
n_{\mathrm{N}, \mathrm{s}}=n_{\mathrm{N}, \mathrm{s}}^{*}\left(\frac{\sigma}{\sigma^{*}}-1\right)\left(\frac{c_{0}}{c^{*}}\right)^{k-1} \exp \left[-\left(\frac{c_{0}}{c^{*}}\right)^{k}\right] \text {. }
$$

According to these data, a characteristic nucleation rate of microcrack density on sectional surface $n_{\mathrm{N}, \mathrm{s}}^{*}$ is calculated as $223 /\left(\mathrm{mm}^{2} \mu \mathrm{m} \mu \mathrm{s}\right)$, according to the following integration:

$$
N_{\mathrm{N}, \mathrm{s}}=\int_{0}^{\infty} n_{\mathrm{N}, \mathrm{s}} \mathrm{d} c_{0}=n_{\mathrm{N}, \mathrm{s}}^{*} \frac{c^{*}}{k}\left(\frac{\sigma}{\sigma^{*}}-1\right) .
$$


5. The transformations from sectional to volumetrical distribution. In our impact tests of the Al alloy, due to the uniaxial strain state and the transverse isotropy, the microcracks are approximately parallel to each other and penny-shaped, also see [3]. For these randomly distributed microcracks, the transformations from sectional to volumetrical distribution are

$$
\begin{aligned}
& N(c)=\int_{c}^{\infty} n\left(c^{\prime \prime}\right) \mathrm{d} c^{\prime \prime}=\frac{2}{\pi} \int_{c}^{\infty} \frac{n_{\mathrm{s}}\left(c^{\prime}\right)}{\sqrt{\left(c^{\prime 2}-c^{2}\right)}} \mathrm{d} c^{\prime}, \\
& n(c)=\frac{2}{\pi} \int_{c}^{\infty} \frac{c}{\sqrt{c^{\prime 2}-c^{2}}}\left[\frac{n_{\mathrm{s}}\left(c^{\prime}\right)}{c^{\prime 2}}-\frac{1}{c^{\prime}} \frac{\mathrm{d} n_{\mathrm{s}}\left(c^{\prime}\right)}{\mathrm{d} c^{\prime}}\right] \mathrm{d} c^{\prime},
\end{aligned}
$$

where $n_{\mathrm{s}}\left(c^{\prime}\right)$ is the number density of microdamages with size $c^{\prime}$ on sectional surface. $n$ and $N$ are the number density of microdamages and the cumulated number density of microdamages greater than $c$, respectively, for more details of (6) and (7) see Appendices A and B in [11]. In fact, both spherical voids and parallel penny-shaped cracks follow the same Eqs. (6) and (7). Clearly, the sectional distribution is usually different from the volumetrical one. But, for the case we described here (Weibull distribution with $k=2.33$ ), they are quite similar in their shapes, Fig. 2.

6. So, substitution of $n_{\mathrm{s}}\left(c^{\prime}\right)=n_{\mathrm{N}, \mathrm{s}}$ and integration of (7) gives the nucleation rate of microcrack density $n_{\mathrm{N}}$ as

$$
\begin{aligned}
& n_{\mathrm{N}}\left(c_{0}, \sigma\right)=n_{\mathrm{N}}^{*}\left(\frac{\sigma}{\sigma^{*}}-1\right) F\left(\bar{c}_{0}\right), \\
& F\left(\bar{c}_{0}\right)=\frac{2}{\pi} \int_{\bar{c}_{0}}^{\infty} \frac{\bar{c}_{0}}{\sqrt{x^{2}-\bar{c}_{0}^{2}}} \xi(x) \mathrm{d} x \\
& \xi(x)=x^{k-3}\left(2-k+k x^{k}\right) \exp \left(-x^{k}\right),
\end{aligned}
$$

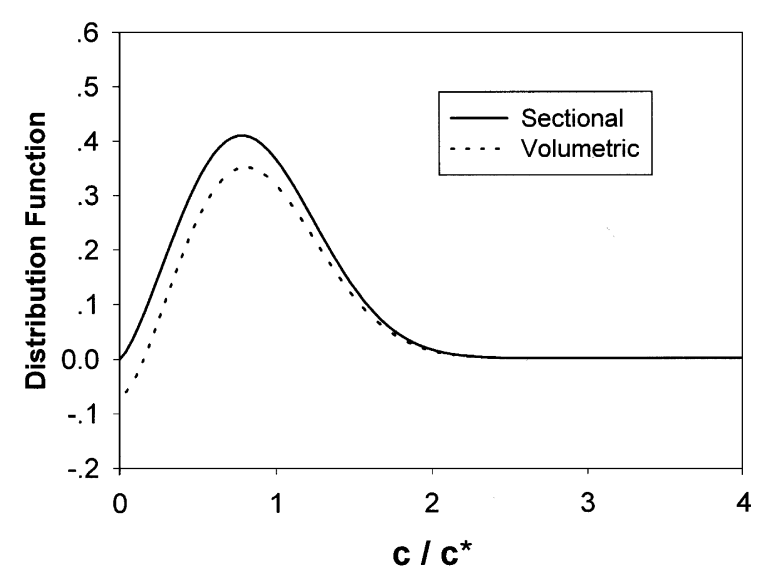

Fig. 2. Comparison of sectional and volumetric distributions (Weibull sectional distribution, with Weibull module $k=2.33)$. 
where $\bar{c}_{0}=c_{0} / c^{*}$ and the characteristic nucleation rate of microcrack density $n_{\mathrm{N}}^{*}=$ $n_{\mathrm{N}, \mathrm{s}}^{*} / c^{*}=5.22 \times 10^{4} /\left(\mathrm{mm}^{3} \mu \mathrm{m} \mu \mathrm{s}\right) . n_{\mathrm{N}}^{*}$ is one of the most important parameters of microdamage evolution in the material.

7. Apart from the nucleation rate, all other mesoscopic dynamic informations involved in the experimental data (such as coalescence) are included in the growth rate of microcracks $C=\dot{c}$, when data fitting were carried out by means of the statistical equation of microdamage evolution [11]. The fitting gives the following formula,

$$
C\left(c, c_{0} ; \sigma\right)=C g \cdot g(\sigma)\left(\frac{c-c_{0}}{c^{*}}\right)^{\mu},
$$

where $\mu=0.775$ and $C g \cdot g(\sigma)=C^{*}=8.1 \mathrm{~m} / \mathrm{s}$ at $1470 \mathrm{MPa}$ in the case study.

Above all, the nucleation rate (8) and growth rate (11) of microcracks are the essential dynamics of microcracks of the Al alloy under one-dimensional strain state impact. Then, what is their link to macroscopical damage evolution and spallation?

Before going into the details of the present study, it might be helpful to have a look at the difference between the prediction made by traditional fracture mechanics and the experimental observation. One can estimate the critical crack size as $c_{\mathrm{c}} \sim K_{I C}^{2} /\left(\pi \sigma^{2}\right)$. When $K_{I C} \sim 30 \mathrm{MPa} \mathrm{m}^{1 / 2}$ and $\sigma=1470 \mathrm{MPa}$, then $c_{\mathrm{c}} \sim 100 \mu \mathrm{m}$. But, from the growth law of microcracks (11), one can integrate it to obtain the time $t_{\mathrm{c}}$ to attain the critical size $c_{\mathrm{c}}$,

$$
t_{\mathrm{c}}=\frac{c^{*}}{(1-\mu) C^{*}}\left[\frac{c_{\mathrm{c}}-c_{0}}{c^{*}}\right]^{(1-\mu)} .
$$

Assuming $c_{0}=c^{*}$ and substituting the values of $c^{*}, C^{*}$ and $\mu$ into the expression, we obtained the time $t_{\mathrm{c}}$ for a microcrack to grow to the critical size $c_{\mathrm{c}}$ is about $4 \mu \mathrm{s}$. In all the tests of the Al alloy, there are no specimens, which can survive beyond $1 \mu$ s under $1470 \mathrm{MPa}$. Furthermore, no individual cracks with such a length were observed in the impact tests. So, we suppose that there should be another mechanism relevant to collective interaction between microdamages governing the failure in spallation.

\section{Inversion to evolution law of damage}

Actually, the observations of microcrack evolution outlined in last section provide a statistical description of number density of microcracks $n_{0}\left(t, c, c_{0} ; \sigma\right)$, where $c$ and $c_{0}$ are the current and nucleation sizes of microcracks, respectively. According to statistical microdamage mechanics, there is a statistical equation of microdamage evolution, Eq. (1). For a representative volume element, the equation can be reduced to a equation governing the evolution of number density of microcracks $n_{0}$ with independent variables $t, c$ and $c_{0}$,

$$
\frac{\partial n_{0}}{\partial t}+\frac{\partial\left(n_{0} C\right)}{\partial c}=n_{\mathrm{N}} \delta\left(c-c_{0}\right)
$$


where $\delta$ is Dirac $\delta$-function and $C$ is the growth rate of microdamages. Corresponding initial and boundary conditions are

$$
\begin{aligned}
& n_{0}\left(0, c, c_{0} ; \sigma\right)=0, \\
& n_{0}\left(t, c, c_{0} ; \sigma\right)=0, \quad c \leqslant c_{0} .
\end{aligned}
$$

Now, we define the $\kappa$-order damage moment in terms of the number density of microcracks $n_{0}$ as follows [12]:

$$
D=\alpha \int_{0}^{\infty} \int_{0}^{\infty} c^{\kappa} n_{0} \mathrm{~d} c_{0} \mathrm{~d} c,
$$

where $\alpha$ is a geometric parameter, for spherical microdamage $\alpha=\pi / 6$. When $\kappa=3, D$ is the volumetric fraction of damage. In continuum damage mechanics, continuum damage is defined as the reduction of load-supporting area. For uniformly random microvoids, the sectional fraction of damage, i.e. the continuum damage, is equal to the volumetric fraction of damage according to stereography. So, the above-defined damage moment $D$ with $\kappa=3$, Eq. (15), is actually the continuum damage. For the penny-shaped microcracks formed in the $\mathrm{Al}$ alloy subjected to plate impact, we assume that there is a stress release volume surrounding each microcrack. So, the damage moment $D$ is approximately the continuum damage in the examined case too.

Now, we are able to compute the evolution of continuum damage $D$, by integrating Eq. (12) and calculating the integration (15) simultaneously, when the mesoscopic dynamics of nucleation and growth of microdamages are known. To facilitate computation, we adopted an assumption of $g(\sigma)$,

$$
g(\sigma)=\left(\frac{\sigma}{\sigma^{*}}-1\right)
$$

Before going to the numerical result of the evolution of continuum damage $D$, we should introduce a characteristic damage $D^{*}=\alpha n_{\mathrm{N}}^{*} c^{* 5} / C^{*} \sim 3.09 \times 10^{3}$. This indicates how much the magnitude of continuum damage would become during the characteristic period of $t^{*}=c^{*} / C^{*} \sim 0.527 \mu \mathrm{s}$. In the following calculations, we always use dimensionless variables, for example, the dimensionless time $t$ is defined by the quotation of real time over the characteristic time $t^{*}$ and the dimensionless stress $\sigma$ by threshold stress $\sigma^{*}$.

Another issue worth mentioning is that the above approach to the evolution law of damage is based on the statistical equation of microdamage evolution. There is no need for any assumptions of potential function or empirical form of evolution law of damage.

Fig. 3 shows the evolution law of continuum damage, i.e. $\dot{D}$ versus $D$, obtained from the integration of Eq. (12) and the integral (15), in the case study with nominal tensile stress $1470 \mathrm{MPa}$.

\section{Dynamic function of damage}

Now we go on to the damage field and the dynamic function of damage. According to the statistical microdamage mechanics, when we turn the equation of number density of microdamage 


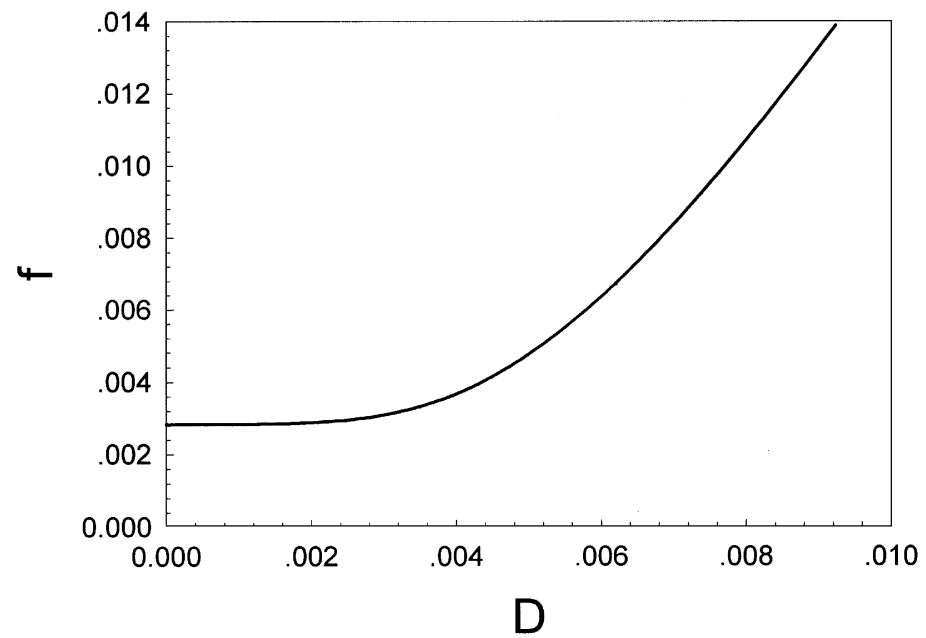

Fig. 3. The evolution law of continuum damage, $\dot{D} \approx f$ versus $D$, obtained from the integration of Eq. (12) and the integral (15), with nominal tensile stress $1470 \mathrm{MPa}$ and dynamics of microcracks, Eqs. (8) and (11). $\dot{D}$ and $f$ are dimensionless by characteristic time $t^{*}$.

$n$, Eq. (1), into the equation of damage moment $D$, there is a dynamic function of damage (DFD) $f$ defined as follows $[14,15]$ :

$$
f=\int_{0}^{\infty} n_{\mathrm{N}} \tau \mathrm{d} c+\int_{0}^{\infty}\left(n A \tau^{\prime}-n_{\mathrm{A}} \tau\right) \mathrm{d} c,
$$

where $\tau^{\prime}=\partial \tau / \partial c, \tau$ being the average failure volume of a microdamage. This function includes all mesoscopic dynamics of microdamage, such as, nucleation and annihilation rate densities of microdamage $n_{\mathrm{N}}$ and $n_{\mathrm{A}}$, the average growth rate of microdamage $A=\int C\left(c, c_{0}\right) n_{0} \mathrm{~d} c_{0} / \int n_{0} \mathrm{~d} c_{0}$.

In this way, the damage field evolution equation, continuum and momentum equations describe the evolution of deformation and damage field. In Lagrangian coordinates in one-dimensional strain state, they are as follows $[14,15]$ :

$$
\begin{aligned}
& \frac{\partial D}{\partial T}+D \frac{V_{0}}{V} \frac{\partial v}{\partial Y}=f, \\
& \frac{\partial V}{\partial T}-V_{0} \frac{\partial v}{\partial Y}=0 \\
& \frac{\partial v}{\partial T}-V_{0} \frac{\partial \sigma}{\partial Y}=0
\end{aligned}
$$

where $V_{0}$ and $V$ are the initial and current specific volumes of a continuum element, respectively. $v$ and $\sigma$ are the particle velocity and the stress of continuum element, respectively. 
We define a dilatation rate

$$
\dot{\theta}=\frac{V_{0}}{V} \frac{\partial v}{\partial Y} .
$$

Actually, the term of $D \dot{\theta}$ in the damage field equation is the difference between the evolution rate of continuum damage $\dot{D}$ and the dynamic function of damage $f$. When damage $D \ll 1$, this term might be much less than the dilatation rate $\dot{\theta}$ and then less than the evolution rate of continuum damage $\dot{D}$, if these two rates are in the same order. So, as an approximation, we can take the above obtained evolution rate of damage $\dot{D}$ as the dynamic function of damage (DFD) $f$.

$$
f=f(D, \sigma) \approx \dot{D} .
$$

Fig. 3 shows the dimensionless DFD $f$.

The experimental results outlined in Section 2 show that for the concerned spallation the dynamic function of damage can be fitted to a binary function of damage $D$ and stress $\sigma$, as suggested by Davison and Steven [2],

$$
f=f(D, \sigma)=G(\sigma) \gamma\left(1+\beta D^{m}\right)
$$

where $G$ is a dimensionless function of stress. $\gamma$ is a dimensionless parameter denoting the relative nucleation rate. $m$ and $\beta$ are two parameters relevant to damage growth and coalescence. Davison and Stevens have named the two terms in (22) as simple and compound damages, respectively [2]. Now, it is clear that the simple and compound damages are rooted to nucleation and growth (as well as coalescence) of microdamage, respectively. Generally speaking, the DFD has $m>1$ and appears to be concave. In particular, $\beta$ is the ratio of compound rate over nucleation rate of damage, so it is an intrinsic Deborah number.

Fig. 4 demonstrates some fittings of DFD (22) in different ranges of damage, such as $D^{*}, 2 D^{*}$ and $3 D^{*}$, under tensile stress $1470 \mathrm{MPa}$. It can be seen that the fittings are all quite well in comparison with the original calculated data. For the convenience of the following calculation, the fitting parameters and their standard deviations (in parenthesis) are given in Table 1.

Before going further, let us have a close look at these fitting parameters. Firstly, the introduction of the dynamic function of damage (DFD), for example formula (22), reduces a number of mesoscopic parameters governing microdamage evolution, at least the six $\sigma^{*}, c^{*}, k, n_{\mathrm{N}}^{*}, C^{*}$ and $\mu$, into three $D^{*}$ (or $\left.G \gamma\right), m$ and $\beta$. This can simplify the formulation of damage evolution in engineering. The value of $G \gamma \sim(2.7-2.8) \times 10^{-3}$ means that the damage due to nucleation attained during the period of characteristic time $t^{*}$ is a bit less than the characteristic damage $D^{*} \sim 3 \times 10^{3}$. Index $m \sim(2.5-2.8)$ reflects the curvature of compound damage. More importantly, the intrinsic Deborah number $\beta$ is as high as $10^{5}-10^{6}$. This implies that the compound rate can become higher than the nucleation rate around the characteristic damage $D^{*}$. So, for further development of damage, compound damage is truly decisive.

\section{Damage localization in spallation}

It has been found that damage localization may occur beyond a threshold and leads to eventual failure. Instead of the complete derivation of the threshold, see $[14,15]$, here we 


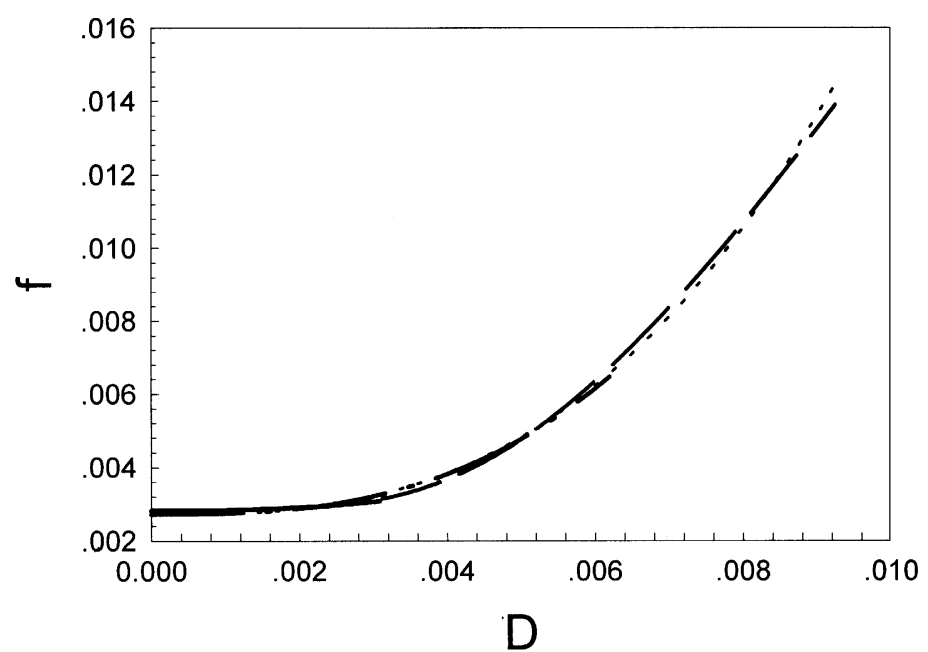

Fig. 4. Fittings of DFD $f$ in different ranges of damage, $D^{*}, 2 D^{*}$ and $3 D^{*}$, with Eq. (22), under tensile stress $1470 \mathrm{MPa}$ $(\ldots \ldots)$ original calculated; $(\longrightarrow) D_{\max }=D^{*} ;(-\cdot-) D_{\max }=2 D^{*} ;(---) D_{\max }=3 D^{*}$.

Table 1

Fitting parameters and their deviations of DFD in Eq. (22)

\begin{tabular}{llll}
\hline$D_{\max }$ & $D^{*}$ & $2 D^{*}$ & $3 D^{*}$ \\
\hline$G \gamma$ & $2.818 \times 10^{-3}$ & $2.719 \times 10^{-3}$ & $2.709 \times 10^{-3}$ \\
& $\left(2.4 \times 10^{-6}\right)$ & $\left(1.0 \times 10^{-5}\right)$ & $\left(1.5 \times 10^{-5}\right)$ \\
$m$ & 2.552 & 2.814 & 2.786 \\
$\beta$ & $\left(4.1 \times 10^{-3}\right)$ & $\left(2.2 \times 10^{-3}\right)$ & $\left(2.1 \times 10^{-2}\right)$ \\
& $2.282 \times 10^{5}$ & $2.246 \times 10^{6}$ & $2.010 \times 10^{6}$ \\
& $\left(8.9 \times 10^{2}\right)$ & $\left(5.6 \times 10^{3}\right)$ & $\left(2.0 \times 10^{5}\right)$ \\
\hline
\end{tabular}

cite a simple lower bound to damage localization only. The lower bound can be easily explained as follows.

Actually, once damage localization occurs, the damage in a local area is great, especially its gradient becomes even greater. This implies that a characteristic relative length scale of damage variation becomes shorter. According to this, we assume the reciprocal of relative gradient of damage $\partial D / \partial Y / D$ as such a length scale.

So, suppose that damage localization would occur, if the increase of the relative gradient of damage becomes positive. That is to say,

$$
\frac{\partial}{\partial T}[\partial D / \partial Y / D]>0
$$

where $T$ and $Y$ are Lagrangian temporal and spatial co-ordinates, respectively. Inequality (23) can be rewritten as

$$
\left(\frac{\partial(\partial D / \partial Y)}{\partial T}\right) /\left(\frac{\partial D}{\partial Y}\right)>\left(\frac{\partial D}{\partial T}\right) / D .
$$




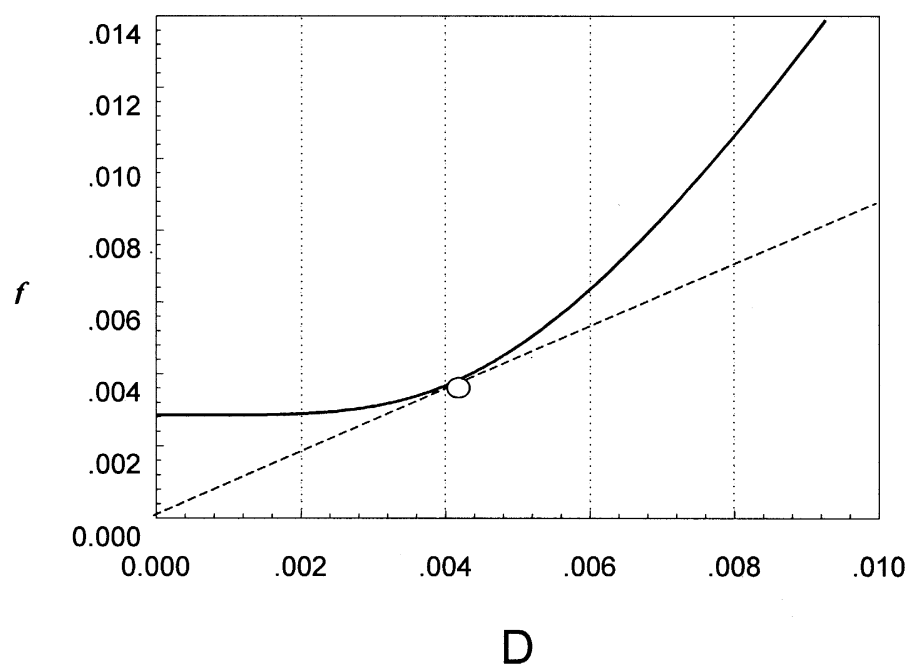

Fig. 5. A sketch of the lower bound to damage localization. The solid line is the DFD and the dashed line indicates the coincidence of the gradient of DFD with respect to damage $f_{\mathrm{D}}$ and its secant $f / D$. The circle $\mathrm{O}$ denotes the critical point, beyond which damage localization may occur.

Substitution of the approximate expression of DFD (21) into the inequality (24) gives

$$
f_{\mathrm{D}}+f_{\sigma} \frac{(\partial \sigma / \partial Y)}{(\partial D / \partial Y)}>\frac{f}{D}
$$

Under quasistatic approximation, namely ignoring inertial term in Eq. (20), the Equation leads to $\partial \sigma / \partial Y=0$, i.e. $\sigma=\sigma(T)$ only. Then, the vanishing stress gradient will lead to a simple lower bound to damage localization,

$$
f_{\mathrm{D}}>\frac{f}{D}
$$

This means that heterohomogeneous damage distribution may evolve to localization when the gradient of the DFD with respect to damage itself $f_{\mathrm{D}}$ becomes greater than its secant $f / D$. Actually, the quasistatic assumption, i.e. ignoring inertia effect, does lead to a lower bound, because inertia retards damage localization, [14]. The lower bound to damage localization is sketched in Fig. 5. Moreover, the damage localization appears to be a real dynamo for material failure. This looks like the well-known Chapman-Jouguet condition for detonation front [17]. Because of the concavity of DFD, damage localization perhaps is a very common phenomenon.

Now, we apply the lower bound to damage localization (25) to the case of spallation. For the expression of DFD (22) deduced from the data of the mesoscopical observations in spallation, this lower bound leads to a simple formula to calculate the critical damage

$$
D_{\mathrm{c}}=[(m-1) \beta]^{-1 / m} .
$$


Table 2

Critical damage $D_{\mathrm{c}}$, predicted life $T_{\mathrm{Dc}}$ and $T_{1}$ as well as ratio of $T_{\mathrm{Dc}} / T_{1}$

\begin{tabular}{lllll}
\hline$D_{\max }$ & $D^{*}$ & $2 D^{*}$ & $3 D^{*}$ & Original data \\
\hline$G \gamma$ & $2.818 \times 10^{-3}$ & $2.719 \times 10^{-3}$ & $2.709 \times 10^{-3}$ & \\
$m$ & 2.552 & 2.814 & 2.786 & \\
$\beta$ & $2.282 \times 10^{5}$ & $2.246 \times 10^{6}$ & $2.010 \times 10^{6}$ & \\
$D_{\mathrm{c}}$ & $6.49 \times 10^{-3}$ & $4.48 \times 10^{-3}$ & $4.44 \times 10^{-3}$ & $4.03 \times 10^{-3}$ \\
$T_{\mathrm{Dc}}$ & 2.01 & 1.46 & 1.45 & 1.35 \\
$T_{1}$ & $(1.06 \mu \mathrm{s})$ & $(0.77 \mu \mathrm{s})$ & $(0.77 \mu \mathrm{s})$ & $(0.71 \mu \mathrm{s})$ \\
$T_{\mathrm{Dc}} / T_{1}$ & 3.68 & 2.53 & 2.52 & 2.52 \\
\hline
\end{tabular}

The lower bound (26) gives a critical damage $D_{\mathrm{c}}=4.03 \times 10^{-3}$ for the case study of spallation. Very importantly, this critical damage is an intrinsic material quantity. Noticeably, $\beta$ denotes the competition of growth and coalescence over nucleation of microdamage. Table 2 gives the calculated critical damage $D_{\mathrm{c}}$ in different fitting ranges $D_{\max }$. Another significant aspect can be seen from the comparison of the three quantities, the critical damage $D_{\mathrm{c}}=4.03 \times 10^{-3}$, the characteristic damage $D^{*} \sim 3 \times 10^{3}$ and $G \gamma \sim(2.7-2.8) \times 10^{-3}$ the damage due to nucleation during $t^{*}$. Once again, the minor difference between $D_{\mathrm{c}}$ and $G \gamma$ (and $D^{*}$ ) clearly shows that the growth and coalescence of microdamage, i.e. the compound damage, plays a key role in damage localizatiom and then in the eventual spallation.

From the definition of DFD (21), we can go ahead to calculate the life of specimen as

$$
T=\int \frac{\mathrm{d} D}{f} .
$$

When adopting the expression of DFD (22), we can calculate the life of materials due to damage localization, in terms of the following formula:

$$
T_{D_{\mathrm{c}}}=\frac{1}{G \gamma} \int_{0}^{D_{\mathrm{c}}} \frac{\mathrm{d} D}{\left(1+\beta D^{m}\right)} .
$$

We may also calculate the maximum life $T_{1}$ by extrapolating (21) to the upper limit $D=1$ in the integral (27) as continuum damage mechanics usually do,

$$
T_{1}=\frac{1}{G \gamma} \int_{0}^{1} \frac{\mathrm{d} D}{\left(1+\beta D^{m}\right)} .
$$

Clearly, the predicted life $T_{1}$ can be taken as the upper limit of life of the material under the same loading. Both predictions $T_{\mathrm{Dc}}$ and $T_{1}$ are listed in Table 2. Corresponding real-time lives, i.e. those multiplied by the characteristic time $t^{*} \sim 0.527 \mu$ s, are included in brackets too. It can be seen that the time to damage localization $T_{\mathrm{Dc}}$ is about $0.7 \mu \mathrm{s}$ but the upper limit of life $T_{1}$ is greater than $1 \mu \mathrm{s}$. But, in reality, the longest duration of the test is $0.8 \mu \mathrm{s}$. 
Table 3

Critical damage $D_{\mathrm{c}}$, predicted lives $T_{\mathrm{Dc}}$ and $T_{1}$ as well as ratio of $T_{\mathrm{Dc}} / T_{1}$, calculated within shorter ranges of damage $D_{\max }$

\begin{tabular}{lllll}
\hline$D_{\max }$ & $0.8 D^{*}$ & $0.6 D^{*}$ & $0.4 D^{*}$ & Original data \\
\hline$G \gamma$ & $2.823 \times 10^{-3}$ & $2.832 \times 10^{-3}$ & $2.834 \times 10^{-3}$ & \\
$m$ & 2.404 & 2.282 & 2.121 & \\
$\beta$ & $6.336 \times 10^{4}$ & $1.677 \times 10^{4}$ & $2.405 \times 10^{3}$ & \\
$D_{\mathrm{c}}$ & $8.73 \times 10^{-3}$ & $1.3 \times 10^{-2}$ & $2.4 \times 10^{-2}$ & $4.03 \times 10^{-3}$ \\
$T_{\mathrm{Dc}}$ & 2.63 & 3.72 & 6.88 & 1.35 \\
$T_{1}$ & $(1.38 \mu \mathrm{s})$ & $(1.96 \mu \mathrm{s})$ & $(3.63 \mu \mathrm{s})$ & $(0.71 \mu \mathrm{s})$ \\
$T_{\mathrm{Dc}} / T_{1}$ & 4.81 & 6.96 & 13.2 & 2.52 \\
& $(2.54 \mu \mathrm{s})$ & $(3.67 \mu \mathrm{s})$ & $(6.97 \mu \mathrm{s})$ & $(1.33 \mu \mathrm{s})$ \\
\hline
\end{tabular}

Form the above results, we can notice that the damage accumulation before the lower bound to localization possesses a large portion of the upper limit of life $T_{1}$, roughly half of it, though the critical damage $D_{\mathrm{c}} \approx 4 \times 10^{-3}$ is 4000 th of the upper limit $D=1$ only. So, damage localization does be able to serve as a processor to failure. Interestingly, in experiments we did scarcely recover and observe damaged but not failured specimens beyond the predicted loading duration of damage localization. Other similar tests confirm the calculations. For example, the tests of the same material under tensile stresses between $1125-1345 \mathrm{MPa}$ and loading durations of $0.68-0.85 \mu$ s do demonstrate microcracks or incipient macro-cracking, see [7]. Longer loading duration leads to complete fracture. These data provide another evidence that the process beyond damage localization is too fast to observe in experiments.

We asked ourselves if we could predict the damage localization or the life of material according to its early data of damage evolution. In order to clarify this point, we carried out the following calculations. We used the data of early damage evolution to predict damage localization and life and then to see how accurate we can achieve in predictions in this way. Table 3 demonstrates some of these results. Clearly, when the data of damage evolution are limited to earlier stage, the prediction of damage localization becomes worse. For meaningful prediction of failure, the data relevant to characteristic damage $D^{*}$ seem to be necessary.

\section{Damage localization in creep}

Additionally, we applied the same idea to calculate the life of an Al alloy in creep rupture [18], at the other extreme of loading spectrum, to testify the concept of damage localization. Hayhurst [18] has given a fitting of the evolution law of damage in creep, which can be adopted as the dynamic function of damage (DFD),

$$
f=\chi(\sigma) /(1-D)^{\phi},
$$


where $\phi$ is a parameter and $\chi(\sigma)$ is a function of stress $\sigma$ given in [18]. For comparison, we fit (30) into another DFD expression similar to Eq. (22),

$$
f=G(\sigma)\left(1+\phi D+\beta D^{m}\right) .
$$

We can apply expressions (30) and (31) to the calculations of critical damage $D_{\mathrm{c}}$ for damage localization, via Eq. (25):

$$
D_{\mathrm{c}}=\frac{1}{1+\phi} \text {. }
$$

Noticeably, Eq. (31) does not change the expression (26) of critical damage for damage localization. We can also derive the life $T_{\mathrm{Dc}} / T 1$, via Eqs. (27) and (30),

$$
\frac{T_{\mathrm{Dc}}}{T 1}=1-\left(\frac{\phi}{1+\phi}\right)^{1+\phi} .
$$

Table 4 gives the fittings and predictions for three materials in creep. The first line in the table gives the estimations of the lifes according to Hayhurst's experimental observations. From the values of the life, one can see that the predictions made by the concept of damage localization, Eq. (33) are qualitatively in agreement with the observations. Still, damage localization serves as a nice processor to failure in creep rupture. In addition, the predicted $D_{\mathrm{c}}$ from both fittings (30) and (31) are nearly the same. Now, let us have a close look at the case of Al alloy. The obtained results are $D_{\mathrm{c}} \sim 0.1$ and $T_{\mathrm{Dc}} / T 1 \sim 0.65$, with the fitting parameters $m=2.23$ and $\beta=144$. Compared to the impact tests, the relative difference of the indices $m$ of compound damage $m$ in the two extremes are one quarter only. But, the intrinsic Deborah number $\beta$ of the impact tests is four orders higher than that of creep and then the critical damage is about two orders lower. This reflects the essential difference of the two kinds of tests. Surprisingly, although there is such a great difference in loading time span, the proposed concept of damage localization can still provide a reasonable prediction.

As a general reference, Fig. 6 shows the variation of critical damage $D_{\mathrm{c}}$ with the intrinsic Deborah number $\beta$, when assuming $m=2.7$, according to (26).

Table 4

Critical damage $D_{\mathrm{c}}$, and relative damage localization $T_{\mathrm{Dc}} / T_{1}$ in creep

\begin{tabular}{llll}
\hline & $\mathrm{Al}$ & $\mathrm{Cu}$ & 316 steel \\
\hline$T_{\mathrm{Dc}} / T_{1}$ & & & \\
$\quad \operatorname{Exp}$ & 0.96 & 0.83 & 0.71 \\
$\quad D F D(30)$ & 0.65 & 0.66 & 0.70 \\
$D_{\mathrm{c}}$ & & & \\
$D F D(30)$ & 0.095 & 0.143 & 0.343 \\
$D F D(31)$ & 0.098 & 0.147 & 0.345 \\
$\phi$ & 9.5 & 6.0 & 1.914 \\
$m$ & 2.23 & 2.66 & 2.39 \\
$\beta$ & 144 & 60.8 & 9.12 \\
\hline
\end{tabular}




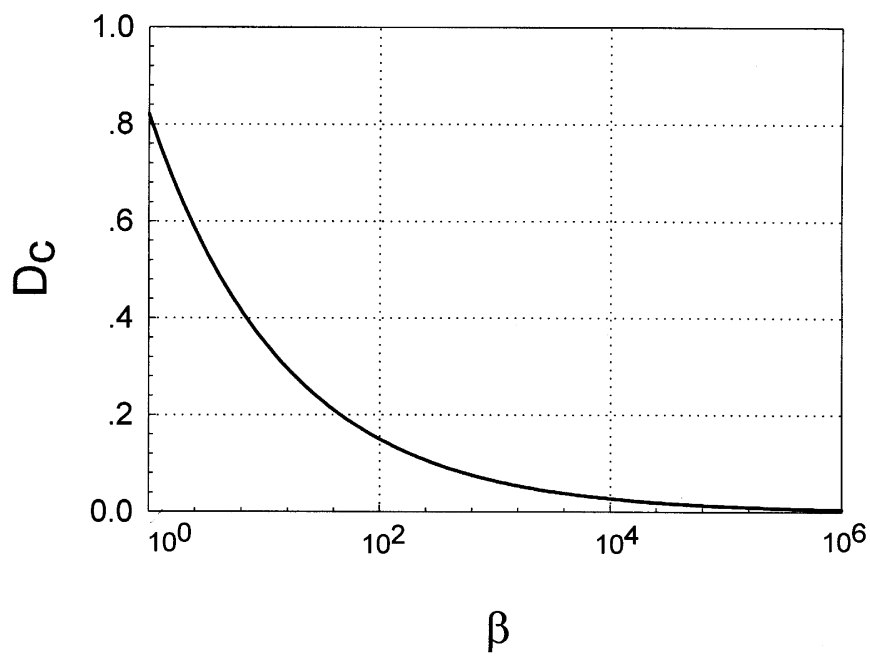

Fig. 6. The variation of critical damage $D_{\mathrm{c}}$ with the intrinsic Deborah number $\beta$, when $m=2.7$, according to Eq. (26).

\section{Conclusions}

A comparative study of statistical microdamage evolution to macroscopic failure was carried out, in order to reveal the correlation between experimentally observed damage evolution and its underlying mesoscopic mechanism.

A dynamic function of damage for an $\mathrm{Al}$ alloy is obtained under plate impact loading, based on relevant experimental measurement of mesoscopic dynamics of microcracks of the alloy as well as the statistical equation of microdamage evolution. The characteristic damage is $D^{*}=\alpha n_{\mathrm{N}}^{*} c^{* 5} / V^{*} \sim 3.09 \times 10^{-3}$. Two parameters of compound damage, the index $m \sim 2.8$ and the intrinsic Deborah number $\beta \sim 2 \times 10^{6}$ play significant role in damage evolution. According to the idea of damage localization leading to failure, a lower bound to damage localization

$$
f_{\mathrm{D}}>\frac{f}{D}
$$

and the corresponding critical damage

$$
D_{\mathrm{c}}=[(m-1) \beta]^{-1 / m}
$$

are derived. The predicted critical damage $D_{\mathrm{c}} \sim 4 \times 10^{-3}$ is a bit greater than the characteristic damage $D^{*}$. The corresponding life $T_{\mathrm{Dc}} \sim 0.77 \mu$ s is supported by experimental observations. When the dynamic function of damage is fitted with longer experimental observations, the calculated critical damage becomes more accurate.

Additionally, the concept of damage localization can also work for the prediction of creep rupture approximately. 


\section{Acknowledgements}

This work is supported by National Natural Science Foundation of China and Chinese Academy of Sciences.

\section{References}

[1] Tuler FR, Butcher BM. A criterion for the time dependence of dynamic fracture. Int J Fract Mech 1968;4:431.

[2] Davison L, Stevens AL. Continuum measures of spall damage. J Appl Phys 1972;43:988-94.

[3] Curran DR, Seaman L, Shockey DA. Dynamic failure of solids. Phys Rep 1987;147:253-388.

[4] Meyers MA. Dynamic behaviour of materials. New York: Wiley, 1994.

[5] Zhou M, Clifton RJ. Dynamic ductile rupture under conditions of plane strain. Int J Impact Engng 1997;19:189-206.

[6] Kawashima K, Nishiura N, Takano M, Nakayama O. Ultrasonic imaging of spall damage under repeated plate impact tests with C-scan acoustic microscope. Rev Prog Quant Nondestructive Eval 17: 1517-22.

[7] Shen LT, Zhao SD, Bai YL, Luo LM. Experimental study on the criteria and mechanism of spallation in an Al alloy. Int J Impact Engng 1992;12:9-19.

[8] Chen D, Al-Hassani STS, Sarumi M, Jin X. Crack straining based spall model. Int J Impact Engng 1997;19:107-16.

[9] Lemanska M, Englman R, Jaeger Z. Transport treatment of crack population in finite medium. Int J Impact Engng 1997;19:257-72.

[10] Bai YL, Ling Z, Luo LM, Ke FJ. Initial development of microdamage under impact loading. ASME Trans J Appl Mech 1992;59:622-7.

[11] Han WS, Xia MF, Shen LT, Bai YL. Statistical formulation and experimental determination of growth rate of micrometre cracks under impact loading. Int J Solids Struct 1997;34:2905-25.

[12] Bai YL, Ke FJ, Xia MF. Formulation of statistical evolution of microcracks in solids. Acta Mech Sinica 1991;7:59-66.

[13] Bai YL, Han WS, Bai J. A statistical evolution equation of microdamage and its application. ASTM STP 1997;1315:150-62.

[14] Bai YL, Xia MF, Ke FJ, Li HL. Damage field equation and criterion for damage localization. In: Wang R, editor. Rheology of bodies with defects. Dordrecht: Kluwer Academic Publishers, 1998. p. 55-66.

[15] Bai YL, Xia MF, Ke FJ, Bai J. Dynamic function of damage and its implications. Key Eng Mater 1998;145-149:411-20.

[16] Han WS, Bai YL. Embryo-damage induced nucleation of microcracks in an aluminium alloy under impact loading. Acta Metall Mater 1995;43:2157-62.

[17] Taylor GI, Tankin RS. In: Emmons HW, editor. Gas dynamics of detonation. Fundamentals of gas dynamics, (Section G). Princeton, NJ: Princeton University Press, 1958.

[18] Hayhurst DR, Dimmer PR, Morrison CJ. Development of continuum damage in the creep rupture of notched bars. Phil Trans R Soc Lond A 1984;311:103-29. 